\title{
Isolation and Characterization of Mouse Testis Specific Serine/Threonine Kinase 5 Possessing Four Alternatively Spliced Variants
}

\author{
Youheng Wei ${ }^{1}$, Guolong $\mathrm{Fu}^{1}$, Hairong Hu${ }^{1}$, Gang Lin ${ }^{1}$, Jingchun Yang ${ }^{1}$, \\ Jinhu Guo ${ }^{2}$, Qiquan $\mathrm{Zhu}^{1}$ and Long Yu${ }^{1, *}$ \\ ${ }^{1}$ State Key Laboratory of Genetic Engineering, Institute of Genetics, School of Life Sciences, Fudan University, \\ Shanghai, 200433, P.R. China \\ ${ }^{2}$ Department of Physiology, UTSouthwestern Medical Center at Dallas, University of Texas, Dallas 75235-9040, TX, USA
}

Received 26 March 2007, Accepted 1 May 2007

\begin{abstract}
Phosphorylation on serine/threonine or tyrosine residues of target proteins is an essential and significant regulatory mechanism in signal transduction during many cellular and life processes, including spermatogenesis, oogenesis and fertilization. In the present work, we reported the isolation and characterization of mouse testis-specific serine/threonine kinase 5 (Tssk5), which contains four alternatively spliced variants including, Tssk5 $\alpha, T_{s s k} \beta \beta$, Tssk5 $\gamma$ and Tssk5 $\delta$. Moreover, the locus of Tssk5 is on chromosome $14 \mathrm{qC} 3$ and the four variants had a similar high expression in the testis and the heart; however, had a low expression in other tissues, except for Tssk5 $\alpha$ which also had comparably high expression in the spleen. Each variant of $T s s k 5$ expression began in the testis 16 days after birth. Aside from TSSK5 $\alpha$, the other isoforms have an insertion of ten amino acid residues (RLTPSLSAAG) in region VIb (HRD domain) (His-Arg-Asp). Moreover, only TSSK5 $\alpha$ exhibited kinase activity and consistently, a further Luciferase Reporter Assay demonstrated that TSSK5 $\beta$, TSSK5 $\gamma$ and TSSK5 $\delta$ cannot be stimulated at the CREB/CRE responsive pathway in emparison to TSSK5 $\alpha$. These findings suggest that TSSK5 $\beta$, TSSK5 $\gamma$, TSSK5 $\delta$ may be pseudokinases due to the insertion, which may damage the structure responsible for active kinase activity. Pull-down assay experiments indicated that TSSK5 $\beta$, TSSK5 $\gamma$ and TSSK5 $\delta$ can directly interact with TSSK5 $\alpha$. In summary, these four isoforms with similar expression patterns may be involved in spermatogenesis through a coordinative way in testis.
\end{abstract}

Keywords: Catalytic motif, HRD (His-Arg-Asp), Phosphorylation, Pseudokinase, Testis specific serine/threonine kinase, TSSK

\footnotetext{
*To whom correspondence should be addressed. Tel: 86-21-65643250; Fax: 86-21-65643250

E-mail: longyu@fudan.edu.cn
}

\section{Introduction}

Protein kinases constitute one of the largest gene families in eukaryotes. By catalyzing the covalent attachment of phosphate onto Ser, Thr or Tyr residues in target proteins, protein kinases regulate a wide variety of conserved cellular processes including, the cell cycle, cell growth, cell death, metabolism, transcription, morphology, motility, and differentiation. In the eukaryotic genome, $2-3 \%$ of genes encode proteins with a kinase domain (Manning et al., 2002; Caenepeel et al., 2004).

Spermatogenesis is a complicated and cyclic process involving specific intercommunication and interaction between the germ and Sertoli cells within the seminiferous tubules, which play a crucial role in the production of functional spermatozoa. Several protein kinases have been revealed involved in the process of spermatogenesis, including Mak, MAST, TESK1 and the testis-specific serine/threonine kinases of the 5 (TSSK) family. Mak (male germ-associated protein kinase) is predominantly expressed in the testicular germ cells during and following meiosis (Matsushime et al., 1990; Jinno et al., 1993). MAST205 (205-kDa manchette microtubuleassociated serine/threonine protein kinase) was identified as the testis-specific protein kinase associated with microtubules in vitro and co-localized with the spermatid manchette. The kinase activity of MAST205 is high in round spermatids and at a maximum in residual bodies, indicating that the MAST205 complex plays a potential function in the spermatid maturation of mammals (Walden et al., 1993; 1996). TESK1 (testisspecific protein kinase), which is most closely related to the LIMK subfamily, is predominantly expressed in testicular germ cells during the late stages from pachytene spermatocytes to round spermatids; however, not in somatic cells such as Sertoli and Leydig cells. The expression pattern of this kinase suggests its key role in spermatogenesis, particularly at the stages of meiosis or early spermatogenesis (Toshima et al., 1995; 1998) 
Testis-specific serine/threonine kinase (TSSK) family members are expressed post-meiotically during spermiogenesis. In addition, the identification of three murine members, tsskl, tssk2 and tssk3 has been reported (Bielke et al., 1994; Kueng et al., 1997; Zuercher at al., 2000; Visconti et al., 2001; Hao et al., 2004). The expression of Tssk1 and Tssk2 was limited to the last stages of spermatid maturation and further immunohistochemical staining further showed the localization of both of these kinases in the cytoplasm of late spermatids and in structures resembling residual bodies; however, absent in released sperms in the lumen of the seminiferous tubules and the epididymis. These results suggest that Tssk1 and Tssk2 likely participate in the construction of cytoplasm during sperm tail maturation. In contrast to Tssk1 and Tssk2, Tssk3 is expressed in Leydig cells surrounding seminiferous tubules, and may have its major function in the differentiated Leydig cells (Zuercher et al., 2000).

Human TSSK5 (testis specific Serine/Threonine kinase 5) plays a role in phosphorylating the transcription factor CREB (cAMP responsive element binding protein) in vitro, and consequently, stimulates the CREB/CRE response pathway in Hek293T cells (Chen et al., 2005). Moreover, Chen et al. (2001; 2005) suggests that hTSSK5 may be involved in spermatogenesis through the phosphorylation of CREB and subsequent stimulation of the CREB/CRE responsive pathway.

In our study, we isolated four mouse testis-specific serine/ threonine kinase 5 (Tssk5) variants which include, $T s s k 5 \alpha$, $T s s k 5 \beta, T s s k 5 \gamma$ and $T s s k 5 \delta$ respectively. The expression patterns in the different tissues and the developmental stages were investigated. Furthermore, the kinase activity and the Luciferase Reporter assay of these Tssk5 variants was assessed and their impact on the CREB/CRE responsive pathway were also carried out and interpreted.

\section{Materials and Methods}

Cloning and identification of mouse TSSK5 and its variants. Mice (ICR line) used in this study were gifts from the Shanghai
Animal Center, China. The four mouse Tssk5 gene variants were isolated from the adult mouse testis cDNA library by PCR amplification using the mtssk5-A and mtssk5-B primer pair. The PCR reaction parameters were: denaturation at $94^{\circ} \mathrm{C}$ for $5 \mathrm{~min}$, annealing at $94^{\circ} \mathrm{C}$ for $60 \mathrm{~s}$ and prolonging at $72^{\circ} \mathrm{C}$ for $60 \mathrm{~s}, 32$ cycles. Through the cloning of the products mixture and subsequent sequencing, four alternatively splicing variants were identified. The PCR products were subcloned into the pMD18-T vector (TaKaRa) and verified by sequencing. The four pairs of primers used to detect the existence and probing the abundance of these variants were designed and synthesized to apply to the PCR reaction (Table 1). The position of the primers were illustrated in Fig. 1. The PCR reaction was: $94^{\circ} \mathrm{C}$ for $5 \mathrm{~min}, 94^{\circ} \mathrm{C}(60 \mathrm{~s}), 64^{\circ} \mathrm{C}(60 \mathrm{~s})$, and $72^{\circ} \mathrm{C}$ $(60 \mathrm{~s})$ for corresponding cycles, with a final extension at $72^{\circ} \mathrm{C}$ for $10 \mathrm{~min}$ in a PE-9600 DNA Engine (Shanghai Fusheng Institute of Biotechnology).

RT-PCR analysis. To investigate the expression abundance of the Tssk5 (SD Comment: As I mentioned above and this applies to rest of text, stick to one way of expressing "Tssk5" and apply it to the whole text) variants and the distribution in different tissues, the total RNA from multiple tissues was isolated with using a single-step isolation method using the TRIzol reagent (Invitrogen) according to the manufacturer's instructions. The cDNA was synthesized using $2 \mathrm{mg}$ of the total RNA, Superscrpt II reverse transcriptase (Invitrogen) and Oligo (dT15) (Promega). The first-strand cDNA was subjected to RT-PCR amplification on the FS-918 DNA Amplifier (Shanghai Fusheng Institute of Biotechnology). We used the corresponding primers (tssk $5 \alpha-\mathrm{F}$, tssk $5 \alpha-\mathrm{R}$, tssk $5 \beta-\mathrm{F}$, tssk $5 \beta-\mathrm{R}$, tssk $5 \gamma-\mathrm{F}$, tssk5 $\gamma-\mathrm{R}$, tssk $5 \delta-\mathrm{F}$, tssk5 $5-\mathrm{R}$ ) of $T s s k 5$ 's and $\beta$-actin to optimize the cycle numbers followed by PCR analysis for 22-34 cycles with $10 \mathrm{ng}$ of the mature mouse cDNA as the template. The PCR reaction cycle of different variants was determined according to the corresponding growth curve of the PCR products. The semiquantitative RT-PCR results were scanned with GDS-800 (BioRad) and analyzed by Annutating Grabber 1T2.51 Scanner software as well as UVP Gelworks ID Advanced software (Version 2.51).

To investigate the expression patterns of $T s s k 5 \mathrm{~s}$ in different developmental stages, new born mice were sacrificed every other day from 8 to 20 days after birth. After the mice have become

Table 1. Primers used in the article

\begin{tabular}{|c|c|}
\hline Primers & Sequence \\
\hline \multicolumn{2}{|c|}{ Primers to clone the cDNAs } \\
\hline$m t s s k 5-A$ & 5'-CACTGCGCAAGCCCAACATCATG-3' \\
\hline$m t s s k 5-B$ & 5'-CCTCCTGCCTCAGCCACTTTCAG-3' \\
\hline \multicolumn{2}{|c|}{ Primers to detect the variants } \\
\hline tssk $5 \alpha-\mathrm{F}$ & 5'-GCAAGGGCATCGTGCACCGGG-3' \\
\hline tssk $5 \alpha-R$ & 5'-CATGCTCCAGGTGTCAGACAGG-3' \\
\hline tssk5 $\beta-\mathrm{F}$ & 5'-AGCCTTTCTGCTGCTGGTAGGG-3' \\
\hline tssk5 $\beta-\mathrm{R}$ & 5'-GCAGCTGGAGGATCAGGTTCTTGCA-3' \\
\hline tssk5 $\gamma-\mathrm{F}$ & 5'-CAGCCTTTCTGCTGCTGGTAGGG-3' \\
\hline tssk $5 \gamma-\mathrm{R}$ & 5'-CCCTGGTTTTATGCTGGAGCCCTTG-3' \\
\hline tssk $5 \delta-\mathrm{F}$ & 5'-GCAAGGGCATCGTGCACCGCC-3' \\
\hline tssk $5 \delta-\mathrm{R}$ & 5'-TTTGAGTAGGAGCTCTGGCATGAC-3' \\
\hline \multicolumn{2}{|c|}{ Primers to generate the mutant } \\
\hline mut-TSSK5-f & 5'-GGTCATGGTGGCTGTCATGATCATCTCGAAGAAGAAGG-3' \\
\hline mut-TSSK5-r & 5'-ССTTCTTCTTCGAGATGATCATGACAGCCACCATGACC-3' \\
\hline
\end{tabular}




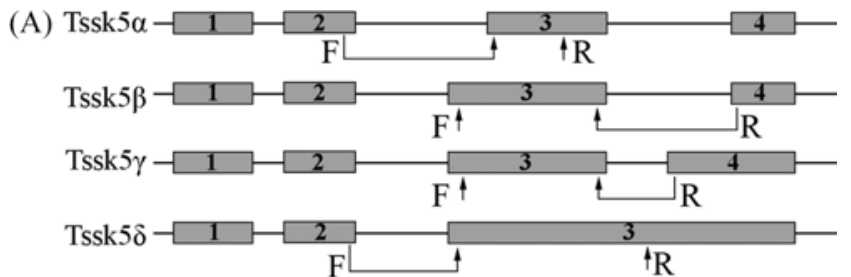

(B)

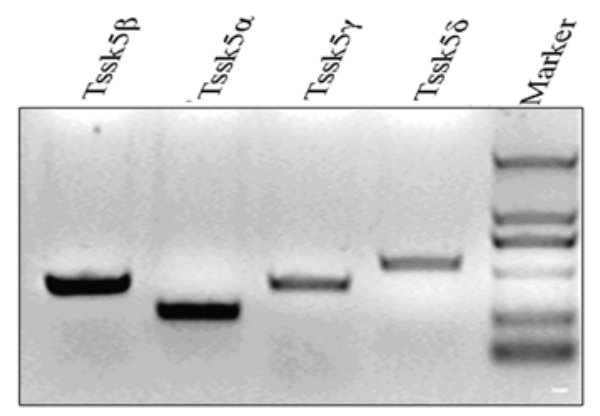

Fig. 1. The exon-intron organization (A) of the $m T s s k 5$ variants and the expression abundance in adult mouse testis (B). Fig. 1A showed different exon-intron organization of the four variants. Boxes shadowed in grey denote the exons, and the connecting lines denote introns. The arrows indicate the position of the primers used in PCR reaction. In Fig. 1B, the upper panel showed the products of RT-PCR and the below panel showed the quantification, correspondingly to each well in the upper panel.

sexually mature at 30 and 60 days, the mice were added to be positive controls.

Plasmid construction and site-directed mutagenesis. The full length fragments of the four mouse TSSK5's were sub-cloned into the eukaryotic expression vector pCMV-Myc within the EcoRI and NotI restriction sites. The Myc-TSSK5 $\alpha-\mathrm{K} 54 \mathrm{M}$ mutant (the corresponding site of the kinase-negative form of human TSSK5) was generated by site-directed mutagenesis using the QuikChange site-directed mutagenesis kit (Stratagene) according to the manufacturer's instructions with the mutation primers mut-TSSK5f and mut-TSSK5-r (Table 1).

Cell culture, transfection and western blotting. HEK293T cells (initially from ATCC) were grown in DMEM medium (Gibco BRL), supplemented with $10 \%$ fetal calf serum (Gibco) (SD Comment: You don't usually need to mention the company's location from the second time the company name is mentioned. It may say otherwise in the instructions to authors of your particular journal, but I just wanted to point it out to you). In addition, $3.5 \times$ $10^{5}$ cells were seeded in $60 \mathrm{~mm}$ dishes. After overnight growth when the cells reached $80 \%$ confluence, transfected the cells with $2 \mu \mathrm{g}$ of mouse TSSK5's plasmid constructs using Lipofectamine ${ }^{\mathrm{TM}}$ Reagent (Invitrogen) in the serum-free DMEM medium. After $5 \mathrm{~h}$ of incubation, the medium was replaced with fresh complete medium, and the cells were cultured for an additional $30 \mathrm{~h}$ prior to harvest and the whole lysate was applied to Western blotting, cMYC monoclonal antibody (Sigma) was used as the primary antibody.
In vitro kinase assays. Trx-His-S-CREB protein was obtained as described in (Chen et al., 2005), [19]. A total of 293T cells were transfected with empty Myc-vector, Myc-TSSK5 $\alpha \mathrm{W}$ (wild type of TSSK $5 \alpha$ ), TSSK $5 \beta$, TSSK $5 \gamma$, TSSK $5 \delta$, Myc-TSSK $5 \alpha-K 54 M$ (mutant of TSSK $5 \alpha$ ), and cell lysates from these cells which were immuno-precipitated by using c-Myc Monoclonal Ab-Agarose beads (CLONTECH) in lysis buffer at $4^{\circ} \mathrm{C}$ for $5 \mathrm{~h}$. The lysis buffer contains: $20 \mathrm{mM}$ Tris- $\mathrm{HCl}$ (pH 7.5), $150 \mathrm{mM} \mathrm{NaCl}, 1 \mathrm{mM}$ $\mathrm{Na}_{2}$ EDTA, 1 mM EGTA, 1\% Triton, $2.5 \mathrm{mM}$ Sodium pyrophosphate, $1 \mathrm{mM}$ glycerophosphate, $1 \mathrm{mM} \mathrm{Na}_{3} \mathrm{VO}_{4}, 1 \mu \mathrm{g} / \mathrm{ml}$ Leupeptin, $1 \mathrm{mM}$ PMSF) and the beads were washed four times with lysis buffer, twice with $1 \times$ kinase assay buffer $(25 \mathrm{mM}$ Tris- $\mathrm{HCl}(\mathrm{pH} 7.5)$, $5 \mathrm{mM}$ glycerophosphate, $2 \mathrm{mM}$ dithionthreitol (DTT), $0.1 \mathrm{mM}$ $\mathrm{Na}_{3} \mathrm{VO}_{4}, 10 \mathrm{mM} \mathrm{MgCl}$ ), and incubated for $30 \mathrm{~min}$ at $30^{\circ} \mathrm{C}$ in $25 \mu \mathrm{l}$ of kinase assay buffer with $200 \mu \mathrm{M}$ ATP, $5 \mathrm{mCi}$ of $\left[\gamma^{32} \mathrm{P}\right] \mathrm{ATP}$ $(3000 \mathrm{Ci} / \mathrm{mmol})$, in the presence of substrate protein with Trx-HisS-CREB and myelin basic protein (MBP) $(0.5 \mathrm{mg} / \mathrm{ml}$, Sigma). The samples were subjected to SDS-PAGE, and ${ }^{32} \mathrm{P}$-labeled proteins were visualized by autoradiography.

Luciferase Reporter Assay. We used the Mercury ${ }^{\mathrm{TM}}$ pathway profiling system (Clontech) and a Dual-Luciferase ${ }^{\circledR}$ reporter assay system (Promega) to assess the signal transduction pathways which could be influenced by the four mouse Tssk5 variants. Hek293T cells were grown in 24-well culture plates $\left(0.8 \times 10^{5}\right.$ cells/well). After $24 \mathrm{~h}$ of growth, $500 \mathrm{ng}$ of plasmid containing $10 \mathrm{ng}$ of the Renilla luciferase reporter vector (Promega) and $100 \mathrm{ng}$ of the mycTSSK5's vectors or the corresponding empty pCMV vector were transfected in triplicate using lipofectinamine ${ }^{\mathrm{TM}}$ (Invitrogen) according to the manufacturer's recommendations. The transfected cells were grown in DMEM with $10 \%$ fetal serum for $28 \mathrm{~h}$ prior to lysis and luciferase detection. Luciferase activity was measured by the DualLuciferase Reporter Assay system (Promega). Moreover, separate luciferase readings were taken for both the reporter plasmid and the Renilla luciferase reporter plasmid.

GST-fusion protein pull-down experiments. Full length TSSK $5 \beta$, $\gamma$ and $\delta$ were cloned into the vector pGEX4T-1 at the EcoRI and NotI sites. The expression and purification of GST fusion proteins was performed according to the protocol of Glutathione Sepharose ${ }^{\mathrm{TM}}$ 4B (Amersham Phamacia Biotech). The purified proteins GST, GST-TSSK $5 \beta, \gamma$ or $\delta$ were covalently attached to the $50 \%$ slurry of glutathione- Sepharose beads, and subsequently incubated with whole-cell lysates from cells expressing Myc-TSSK $5 \alpha$ at $4^{\circ} \mathrm{C}$ for $3 \mathrm{~h}$ in buffer containing $20 \mathrm{mM}$ Tris- $\mathrm{HCl}$ (pH 8.0), $200 \mathrm{mM} \mathrm{NaCl}$, $1 \mathrm{mM}$ EDTA, $0.5 \% \mathrm{NP}-40$ and protease inhibitors. The beads were washed 5 times with a washing buffer containing $20 \mathrm{mM}$ Tris- $\mathrm{HCl}$ (pH 8.0), $500 \mathrm{mM} \mathrm{NaCl}, 1 \mathrm{mM}$ EDTA, 1\% NP-40 and protease inhibitors. Furthermore, the bound proteins were analyzed by immunoblotting by using mouse Myc-specific monoclonal antibody (Sigma).

\section{Results}

Identification the four alternatively spliced variants of mouse Tssk5. Chen et al. (2005) previously reported that the human testis-specific Serine/Threonine kinase TSSK5 could phosphorylate CREB (cAMP responsive element binding 


\begin{tabular}{|c|c|c|}
\hline & II & \\
\hline TSSK5 $\delta$ & MGKGDTSETASATPAYRSVMEEYGYEVGKI IGHGSYGTVYEAYYTKQKVMVAVKIISKKK & 60 \\
\hline TSSK5 $\gamma \gamma$ & MGKGDTSETASATPAYRSVMEEYGYEVGKIIGHGSYGTVYEAYYTKQKVMVAVKI ISKKK & 60 \\
\hline TSSK5 $\alpha$ & MGKGDTSETASATPAYRSVMEEYGYEVGKI IGHGSYGTVYEAYYTKQKVMVAVKIISKKKK & 60 \\
\hline \multirow[t]{3}{*}{ TSSK5 $\beta$} & MGKGDTSETASATPAYRSVMEEYGYEVGKIIGHGSYGTVYEAYYTKQKVMVAVKIISKKK & 60 \\
\hline & 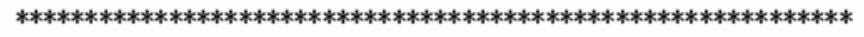 & \\
\hline & IV & \\
\hline SSK5 & ASEDYLNKFLPREIQVMKVLRHKYL INFYQAIETTSRVYIILELAQGGDVLEWIQRYGAC & 120 \\
\hline SSK5 $\gamma$ & ASEDYLNKFLPREIQVMKVLRHKYLINFYQAIETTSRVYIILELAQGGDVLEWIQRYGAC & 120 \\
\hline TSSK5 $\alpha$ & ASEDYLNKFLPREIQVMKVLRHKYLINFYQAIETTSRVYIILELAQGGDVLEWIQRYGAC & 120 \\
\hline \multirow[t]{3}{*}{ TSSK5 $\beta$} & ASEDYLNKFLPREIQVMKVLRHKYLINFYQAIETTSRVYIILELAQGGDVLEW IQRYGAC & 120 \\
\hline & 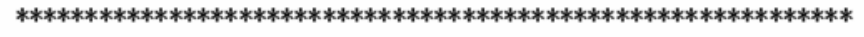 & \\
\hline & $\mathrm{VTb}$ & \\
\hline TSSK5 $\delta$ & 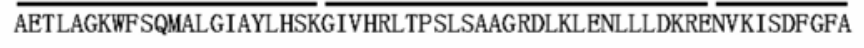 & 180 \\
\hline TSSK5 $5 \gamma$ & AETLAGKWFSQMALGIAYLHSKGIVHRLTPSLSAAGRDLKLENLLLDKRENVKISDFGFA & 180 \\
\hline TSSK5 $\alpha$ & AETLAGKWFSQMALGIAYLHSKGIVH-—-RDLKLENLLLDKRENVKISDFGFA & 170 \\
\hline \multirow[t]{3}{*}{ TSSK5 $\beta$} & AETLAGKWFSQMALGIAYLHSKGIVHRLTPSLSAAGRDLKLENLLLDKRENVK ISDFGFA & 180 \\
\hline & $* * * * * * * * * * * * * * * * * * * * * * * * * * *: \quad: \quad$ & \\
\hline & VIII & \\
\hline TSSK5 $\delta$ & KMVPSSQPVHSSPSYRQMNSLSHLSQTYCGSFAYACPEILLGLPYNPFLSDTWSMGVILY & 240 \\
\hline TSSK5 5 & KMVPSSQPVHSSPSYRQMNSLSHLSQTYCGSFAYACPEILLGLPYNPFLSDTWSMGVILY & 240 \\
\hline TSSK5 $\alpha$ & KMVPSSQPVHSSPSYRQMNSLSHLSQTYCGSFAYACPEILLGLPYNPFLSDTWSMGVILY & 230 \\
\hline \multirow[t]{3}{*}{ TSSK5 $\beta$} & KMVPSSQPVHSSPSYRQMNSLSHLSQTYCGSFAYACPEILLGLPYNPFLSDTWSMGVILY & 240 \\
\hline & $* * * * * * * * * * * * * * * * * * * * * * * * * * * * * * * * * * * * * * * * * * * * * * * * * * * * * * * * * * * * * * * * * *)$ & \\
\hline & $\mathrm{XI}$ & \\
\hline TSSK5 $\delta$ & TLVVARLPFDDTNLKKLLRETQKEVTFPANLTISQECK —-VLAPPGG- & 285 \\
\hline TSSK5 $\gamma$ & TLVVARLPFDDTNLKKLLRETQKEVTFPANLTISQECKGSSIKPGPQP-—_-_-_ & 288 \\
\hline TSSK5 $\alpha$ & TLVVARLPFDDTNLKKLLRETQKEVTFPANLTISQECKNLILQLLRQSTKRATILDVLRD & 290 \\
\hline \multirow[t]{2}{*}{ TSSK5 $\beta$} & TLVVARLPFDDTNLKKLLRETQKEVTFPANLTISQECKNLILQLLRQSTKRAT ILDVLRD & 300 \\
\hline & $* * * * * * * * * * * * * * * * * * * * * * * * * * * * * * * * * * * * * * \quad:$ & \\
\hline SSK5 $\delta$ & 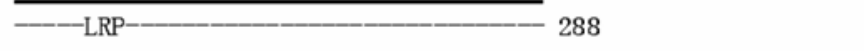 & \\
\hline TSSK5 $\gamma$ & $-{ }_{-}$LSPE-__-_-_ 292 & \\
\hline TSSK5 $\alpha$ & PWMLKFQPEQPSNEIRLLEAMYQPTSSAKRHQSLEITT 328 & \\
\hline TSSK5 $\beta$ & $\begin{array}{l}\text { PWMLKFQPEQPSNEIRLLEAMYQPTSSAKRHQSLEITT } 338 \\
\quad: \star\end{array}$ & \\
\hline
\end{tabular}

Fig. 2. Alignment of the protein sequences of TSSK $5 \alpha, \operatorname{TSSK} 5 \beta, \operatorname{TSSK} 5 \gamma$ and TSSK $5 \delta$. The letters in Greek denote the different functional kinase domains, according to Hanks and Hunters [14-16]. Asterisks denote the consensus sequence of the four variants. The main difference of the four kinases is that TSSK5 $\beta$, TSSK5 $\gamma$ and TSSK5 $\delta$ contain an insertion of ten amino acid residues (RLTPSLSAAG) in region VIb, compared to TSSK5 $\alpha$.

protein) at the Ser133 residue. In the present study, we isolated mouse Tssk5 genes comprising four alternatively spliced variants: $T s s k 5 \alpha, T s s k 5 \beta$, $T s s k 5 \gamma$ and $T s s k 5 \delta$, through the homology cloning strategy. The sequences of $T s s k 5 \alpha, T s k 5 \beta$, $T s s k 5 \gamma$ and $T s s k 5 \delta$ were submitted to GenBank and the deposition numbers are, EF127819, EF127820, EF127821 and EF127822, respectively.

The mouse Tssk5 is located on chr14 qC3 (nt: 5460425354606602), and the transcription of these four variants is attributed to the alternative splicing at the third exon. The third exon of $T s s k 5 \alpha$, in turn, initiated $30 \mathrm{nt}$ downstream of the three other variants (Fig. 1A). To determine their relative expression abundance in adult mouse testis, we designed four pairs of primers and conducted RT-PCR for 27 cycles and RTPCR amplified according to the reaction curve. In addition, the PCR products were also verified by sequencing. The RTPCR revealed that in adult mouse testis, the expression of $T s s k 5 \alpha, T s s k 5 \beta$ was higher in comparison to $T s s k 5 \gamma$ and $\operatorname{Tssk} 5 \delta$ (Fig. 1B).

The proteins of TSSK $5 \alpha$, TSSK $5 \beta$, TSSK $5 \gamma$ and TSSK $5 \delta$ are composed of 328, 338, 292 and 288 amino acid residues, respectively (Fig. 2), and SMART searches (http://smart. embl-heidelberg.de/) indicated that all of them contain only the S TKc (Serine/Threonine protein kinases, catalytic) domain (data not shown, SMART No.: SM00220). We assigned the twelve functional regions in TSSK5's according to the sequence alignment and reveal the amino acids sequences in twelve functional regions (Hanks et al., 1998; 1991a; 1991b; 1995). TSSK5 $\gamma$ and TSSK5 $\delta$ are very short in the C-terminal XI region, due to alternative splicing, and 


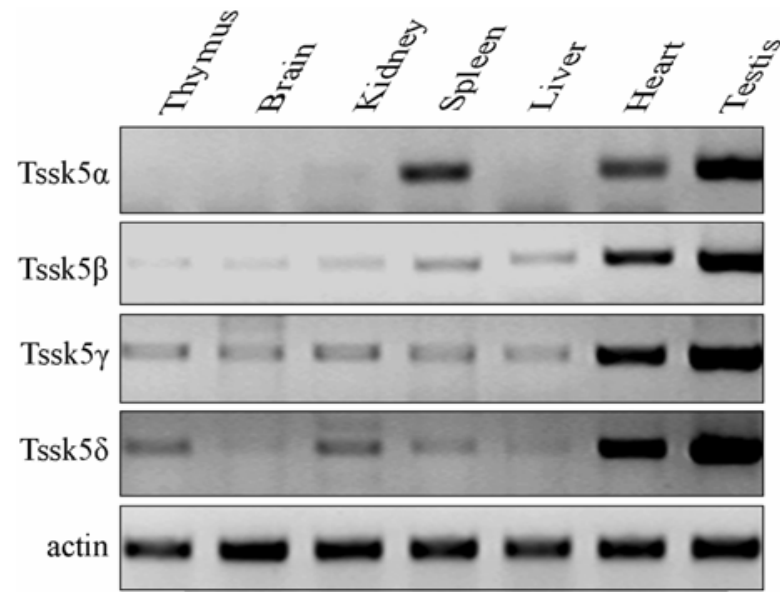

Fig. 3. Tissue distribution of TSSK $5 \alpha$, TSSK $5 \beta, \operatorname{TSSK} 5 \gamma$ and TSSK $5 \delta$ transcripts revealed by RT PCR. All of them were significantly expressed in the testis and heart, although the $\operatorname{TSSK} 5 \alpha$ was also highly expressed in the spleen. $\beta$-actin was used as control.

\begin{tabular}{|c|c|c|c|c|c|c|c|c|}
\hline (Cycles) & 10 & 12 & 14 & 16 & 18 & 20 & 30 & $60 \mathrm{dp}$ \\
\hline Tssk5 $\alpha$ (28) & & & & & & & & \\
\hline Tssk $5 \beta$ (28) & & & & 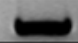 & $\longrightarrow$ & 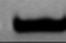 & 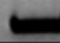 & a \\
\hline Tssk5 $\gamma$ (32) & & 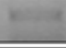 & $5=4$ & 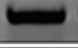 & 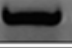 & $\square$ & 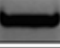 & \\
\hline Tssk5 $(32)$ & $=$ & 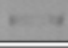 & . & $-\infty$ & $=$ & - & $=$ & (6.) \\
\hline
\end{tabular}

Fig. 4. The different expression patterns of TSSK $5 \alpha$, TSSK5 $\beta$, TSSK $5 \gamma$ and TSSK5 $\delta$ in various developmental stages, revealed by RT-PCR. The TSSK $5 \alpha$ has different developmental pattern to the other three variants. $\beta$-actin was used as control. According to their relative abundance, the PCR cycles of actin, TSSK5 $\alpha$, TSSK $5 \beta, \quad$ TSSK $5 \gamma$ and TSSK $5 \delta$ are 22, 28, 28, 32, 32 respectively. The lanes were from 8 to 30 days of postnatal mouse.

Region VIb of the HRD domain (His-Arg-Asp) forms the catalytic loop (HRD domain) (Manning et al., 2002; Caenepeel et al., 2004; Boudeau et al., 2006). In contrast, compared to TSSK $5 \alpha$, the three other isoforms have a insertion of ten amino acid peptides (RLTPSLSAAG). Moreover, in the HRD domain, the aspartic acid is the catalytic residue, functioning as a base acceptor to achieve proton transfer (Manning et al., 2002; Caenepeel et al., 2004; Boudeau et al., 2006), therefore it's supposed to be crucial for the kinase activity.

The expression patterns of mouse Tssk 5 in different tissues and in testis of different developmental stages. We determined the expression patterns of mouse $T s s k 5$ variants in multiple tissues, including testis, heart, brain, liver, kidney, spleen and thymus (Fig. 3). The results demonstrated that $T s s k 5 \alpha, T s s k 5 \beta$, $T s s k 5 \gamma$ and $T s s k 5 \delta$ showed similar tissues expression, all of them were highly transcribed in testis and heart, and besides,

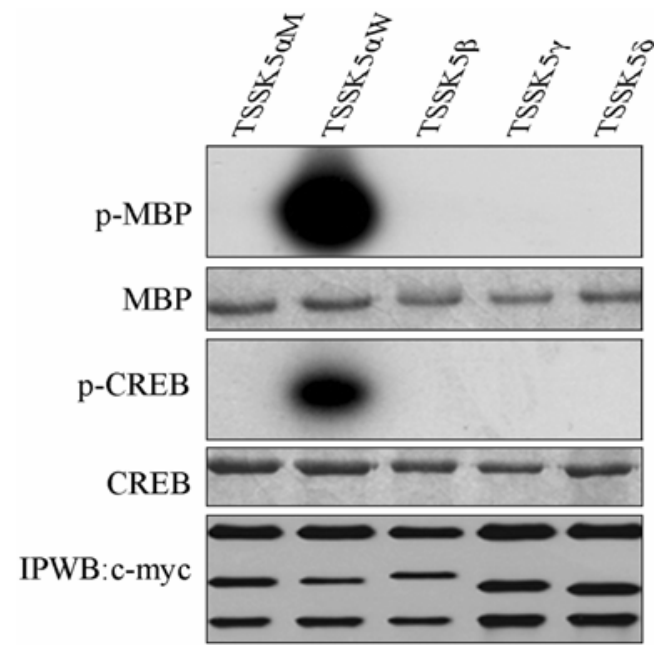

Fig. 5. In vitro kinase assay of TSSK $5 \alpha, \operatorname{TSSK} 5 \beta, \operatorname{TSSK} 5 \gamma$ and TSSK5 $\delta$. Myc-TSSK5 $\alpha \mathrm{M}$ (the mutant of TSSK5 $\alpha$ ), mycTSSK $5 \alpha \mathrm{W}$ (the wild type of TSSK $5 \alpha$ ), myc-TSSK $5 \beta$, mycTSSK $5 \gamma$, myc-TSSK $5 \delta$ were transfected and expressed in the HEK293T cell. The quantities of these genes using in this assay was showed with c-myc monoclonal antibody. MBP and CREB were showed using the Coomassie brilliant blue R250 staining. $\mathrm{P}-\mathrm{CREB}$ and P-MBP were indicated by autoradiography.

$T s s k 5 \alpha$ also showed comparable expression in spleen (Fig. 3). In different developmental stages, all these four variants started to be expressed highly from 16 days postnatal when was recognized as sexually mature. $T s s k 5 \beta, T s s k 5 \gamma$ and $T s s k 5 \delta$ also showed slight expression prior to 16 days, while $T s s k 5 \alpha$ was not detectable until 14 days (Fig. 4). The results suggested that TSSK $5 \alpha$ may play different roles in mouse testis sexual maturation compared to the other three variants.

In vitro Kinase activity of mouse TSSK5's. We performed in vitro kinase assay with His-CREB and MBP as substrates (Fig. 5). Constructs of the four variants were used to transfect Hek293T cells, TSSK5 $\alpha \mathrm{M}$ and pCMV-vector were used as negative control. The kinase assay results indicated that only TSSK $5 \alpha$ exhibited the kinase activity, while the rest three showed no detectable signal (Fig. 5). These results revealed that only TSSK $5 \alpha$ is an active kinase, while TSSK $5 \beta$, TSSK5 $\gamma$ and TSSK $5 \delta$ might be pseudokinases.

Luciferase reporter assay of TSSK5 isoforms. We used the mercury pathway profiling system and a Dual-Luciferase reporter assay system to examine whether these mTSSK5 isoforms can influence the CRE/CREB responsive pathway. The results showed that only TSSK $5 \alpha$ could stimulate pCREluciferase activity compared with the others and the negative control (about 2.5-fold higher from the background) (Fig. 6). These results further showed in vivo that only the TSSK $5 \alpha$ had the kinase activity, TSSK5 $\beta$, TSSK $5 \gamma$ and TSSK $5 \delta$ showed no detectable kinase activity. 


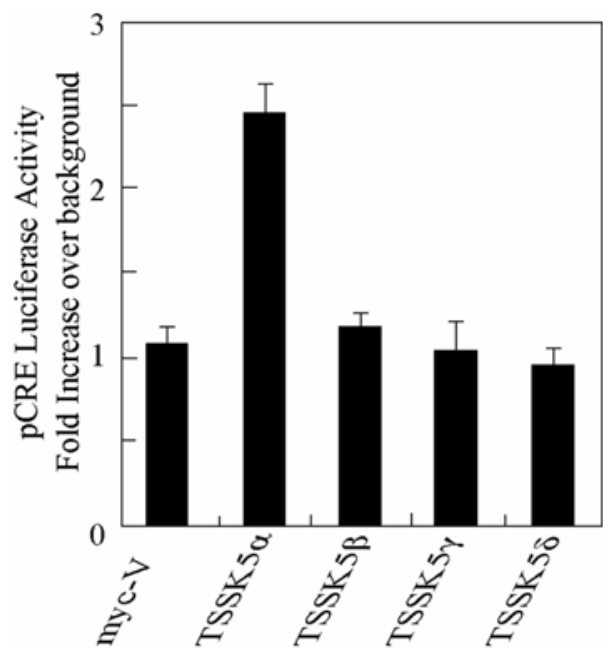

Fig. 6. Luciferase activity assay of TSSK $5 \alpha, \operatorname{TSSK} 5 \beta, \operatorname{TSSK} 5 \gamma$ and TSSK5 $\delta$. Basal expression of cells transfected with empty vector control was normalized to 1 . Error bars represented the mean + S.E. from at least three independent treatments.

\begin{tabular}{|c|c|}
\hline AKT & EDRTRFYGAETVSALDYLHSGKIVY--------RDLKLENLILD \\
\hline PKA & EPHARFYAAQTVL TFEYLHSLDLIY--- \\
\hline RSK & EEDVKFYLAELALGLDHLHSLG IIY---------RDLKPENILLD \\
\hline MSK & EAEVRVYGGETVLALEHLHKLGIIY-- \\
\hline CAMK & EADASHCIQQZEAVLHCHQMGVVH-- \\
\hline TSSK $a$ & ETLAGKWFSQMALGIAYLHSKG IVH-------RDLKLERLLLD \\
\hline TSSK $\beta$ & ETLAGKWF SQMALGIAYLHSKG IVHRL TP SLSAAGRDLKLENLLLD \\
\hline TSSK $\mathrm{x}$ & ETLAGKWF SQMALGIAYLHSKG IVHRL TPSLSAAGRDLKLENLLL \\
\hline ISSK $\delta$ & ETLAGKWFSQMALGIAYLHSKG IVHRL TPSLSAAGRDLKLERLL \\
\hline
\end{tabular}

Fig. 7. The HRD domains of TSSK $5 \alpha$, TSSK $5 \beta$, TSSK $5 \gamma$ and TSSK $5 \delta$ and several Serine/Threonine kinase such as AKT, PKA, RSK, MSK and CAMK were aligned using the CLUSTALW software.

The interaction between TSSK5 $\alpha$ and TSSK5 $\beta$, TSSK5 $\gamma$ and TSSK5 $\delta$. Through GST-pull down experiments we found that TSSK $5 \beta$, TSSK $5 \gamma$ and TSSK $5 \delta$ can directly interact with TSSK $5 \alpha$, respectively (Fig. 8).

\section{Discussion}

The molecular mechanisms regulating the spectacular cytodifferentiation observed during spermiogenesis are poorly understood. The TSSK (Testis Specific Serine/Threonine Kinase) family may play roles in the spermatogenesis and male germ cell maturation, although the mechanisms behind this remain unclear (Bielke et al., 1994; Kueng et al., 1997; Zuercher et al., 2000; Chen et al., 2005). Several members of this family have been identified, including TSSK1, TSSK2, TSSK 3 and TSSK4. In the case of TSSK4, only its sequence was deposited in the GenBank Database (BC111088). No functional annotation has been available as of yet. Ziemiecki et al. (1997, 2000) cloned mouse Tssk1, Tssk2, Tssk3 and found

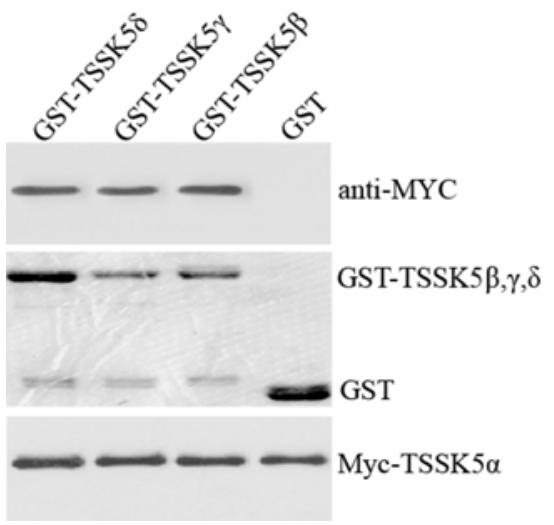

Fig. 8. TSSK $5 \alpha$ interacts with TSSK $5 \beta$, TSSK $5 \gamma$ and TSSK $5 \delta$ in vitro. Whole cell lysates from cells expressing Myc-TSSK $5 \alpha$ protein were incubated with GST, GST-TSSK5 $\beta$, GST-TSSK $5 \gamma$ and GST-TSSK5 $\delta$ fusion proteins respectively. After binding to glutathione-Sepharose beads, washing, and the final bead-bound proteins were detected by Western blotting using MYC monoclonal antibody. The middle panel shows the GST fusion proteins by Coomassie brilliant blue. The lower panel shows the same amount of TSSK $5 \alpha$ protein was used in this assay.

that the expression of Tssk1, Tssk2 and Tssk3 was limited to the final stages of spermatid maturation by immunohistochemical staining (Bielke et al., 1994; Kueng et al., 1997; Zuercher et al., 2000). Chen et al. (2005) cloned human TSSK5 and mapped it to $14 \mathrm{q} 11.2$. As a consequence, in vitro experiments showed hTSSK 5 could phosphorylate the transcription factor CREB (cAMP responsive element binding protein), and stimulate the CREB/CRE responsive pathway in Hek293T cells. This work suggests that CREB is likely one of the important substrates of human TSSK5.

In our present work, we revealed that $T s s k 5 \alpha, T s s k \beta$, $T s s k 5 \gamma$ and $T s s k 5 \delta$ exhibited similar expression patterns in multiple tissues with all of the variants highly expressed in mouse testis and heart. In addition, $T s s k 5 \alpha$ was also expressed highly in spleen. These data suggest that the variants may exert their functions in an efficient manner in testis and heart tissue, but not in the spleen. Consequently, $T s s k 5 \alpha$ may play a role in the independence of $T s s k 5 \beta$, Tssk5 $\gamma$ and $T s s k 5 \delta$. In addition, $T s s k 5 \alpha, T s s k 5 \beta$, Tssk $5 \gamma$ and $T s s k 5 \delta$ started their expression 16 days before an individual reaches sexual maturation, which is exhibited by the potent functions in adult testis (i.e. maintaining the cyclic production of sperm).

Of the known 518 eukaryotic protein kinases encoded by the human genome, 48 have been classified as pseudokinases (Manning et al., 2002). By way of in vitro kinase assay, we demonstrated that mouse TSSK $5 \beta$, TSSK $5 \gamma$ and TSSK $5 \delta$ lack kinase activity. Similarly, TSSK $5 \alpha$ could phosphorylate MBP and CREB (a potent substrate for human TSSK5) (Chen et al., 2005), while TSSK5 $\beta$, TSSK $5 \gamma$ and TSSK $5 \delta$ showed no such activity. This data suggests that TSSK5 $\beta$, TSSK $5 \gamma$ and TSSK $5 \alpha$ may indeed be pseudokinases. 
Compared to TSSK $5 \alpha$ and TSSK5 $\beta$, TSSK $5 \gamma$ and TSSK $5 \delta$ have a very short C-terminus in the XI region; however, TSSK5 $\beta$ also exhibits no detectable kinase activity despite having an identical C-terminus to TSSK $5 \alpha$. This suggests that the XI region is not necessary for kinase activity. Moreover, TSSK $5 \beta$, TSSK $5 \gamma$ and TSSK $5 \delta$ are distinguished by an insertion of 10 (SD Comment: I usually write out numbers under 10, and put the actual number for 10 and above" amino acid residues (RLTPSLSAAG) in region VIb. Furthermore, kinase activity assays demonstrated that TSSK $5 \beta$ TSSK $5 \gamma$ and TSSK $5 \delta$ showed no kinase activity, therefore suggesting that region $\mathrm{VIb}$ is crucial for kinase activity. We have aligned several HRD domains of Ser/Threonine kinase such as AKT, PKA, RSK, MSK and CAMK with the four isoforms and found that they do not contain the 10 amino acid residues (Fig. 7). We speculate that the insertion of the 10 amino acids may connect to the loss of kinase activity of TSSK 5 and as a result, may damage the structure responsible for active kinase activity.

Despite the lack of catalytic activity, pseudokinases may have other important functions. For instance, the pseudokinase (STRAD) regulates the function of the tumor suppressor kinase LKB1 (Baas et al., 2003; Alessi et al., 2006; Boudeau et al., 2004). It has also been reported, that single amino acid substitution within the pseudokinase domain of the tyrosine kinase JAK2 led to several malignant myeloproliferative disorders (Luo et al., 1997; Saharinen et al., 2003). Some pseudokinases have been reported to gain novel roles including, functioning as scaffold instead of kinase activities. For example, TRRAP (a member of the atypical protein kinase PIKK subfamily) possesses an inactive C-terminal pseudokinase domain, which lacks the catalytic residues, suggesting it is a pseudokinase. However, Wang et al., [2005] revealed that TRRAP plays a crucial role in the regulation of chromatin remodeling and gene expression (Wang et al., 2005). As in TSSK5's, since each of the three "pseudokinases" can interact with TSSK5 $\alpha$ (Fig. 8), it's reasonable to speculate that they might play a role in regulating the kinase activity of TSSK $5 \alpha$ in related tissues. Future studies should striveto assess the functional importance and the underlying coordinative mechanism of these four proteins.

Acknowledgments This work was supported by the National 973 Program and 863 High Technology Program of China, as well as the National Natural Science Foundation of China.

\section{References}

Alessi D. R., Sakamoto K. and Bayascas J. R. (2006) LKB1dependent signaling pathways. Annu. Rev. Biochem. 75, 137163.

Baas, A. F., Boudeau, J., Sapkota, G. P., Smit, L., Medema, R., Morrice, N. A., Alessi, D. R. and Clevers, H. C. (2003) Activation of the tumour suppressor kinase LKB1 by the STE20-like pseudokinase STRAD. EMBO. J. 22, 3062-3072.

Bielke, W., Blaschke, R. J., Miescher, G. C., Zurcher, G., Andres,
A. C. and Ziemiecki, A. (1994) Characterization of a novel murine testis-specific serine/threonine kinase. Gene (Amst). 139, 235-239.

Boudeau, J., Saavedra, D. M., Barton, G. J. and Alessi, D. R. (2006) Emerging roles of pseudokinases. Trends in Cell Biol. 16, 443-452.

Boudeau, J., Scott, J. W., Resta, N., Deak, M., Kieloch, A., Komander, D., Hardie, D. G., Prescott, A. R., Van Aalten, D. M. and Alessi, D. R. (2004) Analysis of the LKB1-STRADMO25 complex. J. Cell Sci. 117, 6365-6375.

Caenepeel, S., Charydczak, G., Sudarsanam, S., Hunter, T. and Manning, G. (2004) The mouse kinome: discovery and comparative genomics of all mouse protein kinases. Proc. Natl. Acad. Sci. USA 101, 11707-11712.

Chen, X. J., Lin, G., Wei, Y. H., Hexige, S. Y., Niu, Y. J., Liu, L. L., Yang, C. Y. and Yu, L. (2005) TSSK5, a novel member of the testis-specific serine/threonine kinase family, phosphorylates CREB at Ser-133, and stimulates the CRE/CREB responsive pathway. Biochem. Biophys. Res. Commun. 333, 742-749.

Hanks, S. K. (1991) Eukaryotic protein kinases. Curr Opin. Struct. Biol. 1, 369-383.

Hanks, S. K. and Hunter, T. (1995) The eukaryotic protein kinase superfamily: kinase (catalytic) domain structure and classification. FASEB. J. 9, 576-596.

Hanks S. K. and Quin, A. M. (1991) Protein kinase catalytic domain sequence database: identification of conserved features of primary structure and classification of family members. Methods Enzymol 200, 38-62.

Hanks, S. K., Quin, A. M. and Hunter, T. (1998) The protein kinase family: conserved features and deduced phylogeny of the catalytic domains. Science 241, 42-52.

Hao, Z., Jha, K. N., Kim, Y. H., Vemuganti, S., Westbrook, V. A., Chertihin, O., Markgraf, K., Flickinger, C. J., Coppola, M., Herr, J. C. and Visconti, P. E. (2004) Expression analysis of the human testis-specific serine/threonine kinase (TSSK) homologues. A TSSK member is present in the equatorial segment of human sperm. Mol. Hum. Reprod. 10, 433-444.

Jinno, A., Tanaka, K., Matsushime, H., Haneji, T. and Shibuya, M. (1993) Testis-specific mak protein kinase is expressed specifically in the meiotic phase in spermatogenesis and is associated with a 210-kilodalton cellular phosphoprotein. Mol. Cell. Biol. 13, 4146-4156.

Kueng, P., Nikolova, Z., Djonov, V., Hemphill, A., Rohrbach, V., Boehlen, D., Zuercher G., Andres, A. C. and Ziemiecki, A. (1997) A novel family of serine/threonine kinases participating in spermiogenesis. J. Cell Biol. 139, 1851-1859.

Luo, H., Rose, P., Barber, D., Hanratty, W. P., Lee, S., Roberts, T. M. and Andrea, A. D. (1997) Dearolf C.R., Mutation in the Jak kinase JH2 domain huperactivates Drosophila and mammalian Jak-Stat pathways. Mol. Cell. Biol. 17, 1562-1571.

Manning, G., Whyte, D. B., Martinez, R., Hunter, T. and Sudarsanam, S. (2002) The protein kinase complement of the human genome. Science 298, 1912-1934.

Matsushime, H., Jinno, A., Takagi, N. and Shibuya, M. (1990) A novel mammalian protein kinase gene (mak) is highly expressed in testicular germ cells at and after meiosis. Mol. Cell. Biol. 10, 2261-2268.

Saharinen, P., Vihinen, M. and Silvennoinen, O. (2003) Autoinhibition of Jak2 tyrosine kinase is dependent on specific regions in its pseudokinase domain. Mol. Biol. Cell 14, 1448- 
1459.

Toshima, J., Koji, T. and Mizuno, K. (1998) Stage-specific expression of testis-specific protein kinase 1 (TESK1) in rat spermatogenic cells. Biochem. Biophys. Res. Commun. 249, 107-112.

Toshima, J., Ohashi, K., Okano, I., Nunoue, K., Kishioka, M., Kuma, K., Miyata, T., Hirai, M., Baba, T. and Minzuno, K. (1995) Identification and characterization of a novel protein kinase, tesk1, specifically expressed in testicular germ cells. $J$. Biol. Chem. 270, 31331-31337.

Visconti, P. E., Hao, Z., Purdon, M. A., Stein, P., Balsara, B. R., Testa, J. R., Herr, J. C., Moss, S. B. and Kopf. G. S. (2001) Cloning and chromosomal localization of a gene encoding a novel serine/threonine kinase belonging to the subfamily of testis-specific kinases. Genomics 77, 163-170.

Walden, P. D. and Cowan, N. J. (1993) A novel 205-kilodalton testis-specific serine/threonine protein kinase associated with microtubules of the spermatid manchette. Mol Cell Biol. 13, 7625-7635.

Wang, Z. Q. and Herceg, Z. (2005) Rendez-vous at mitosis: TRRAPed in the chromatin. Cell Cycle 4, 383-387.

Walden, P. D. and Millette, C. F. (1996) Increased activity associated with the MAST205 protein kinase complex during mammalian spermiogenesis. Biol Reprod. Nov. 55, 1039-1044.

Zuercher, G., Rohrbach, V., Andres, A. C. and Ziemiecki, A. (2000) A novel member of the testis specific serine kinase family, tssk-3, expressed in the Leydig cells of sexually mature mice. Mech. Dev. 93, 175-177. 